\title{
Drop Generation due to an Impinging Jet and the Effect of Bottom Blowing in the Steelmaking Vessel
}

\author{
N. STANDISH and Q.L. HE \\ Department of Metallurgy and Materials Engineering, The University of Wollongong, Wollongong, NSW 2500, Australia.
}

(Received on June 13, 1988; accepted in the final form on December 20,1988)

\begin{abstract}
Experimental results of drop generation in a laboratory model water bath with top blowing and combined blowing are given. It was found that with top blowing alone two different mechanisms of drop generation exist, each having its own characteristic drop generation rate. The two mechanisms, identified by high speed film analysis, are shown to be a function of the blowing rate. Bottom blowing is shown to significantly increase drop generation rate and that the increase is caused by the interaction of the top blowing and the bottom blowing in the impingement zone, and not by the bottom blowing as such. Drop size distribution generated by a gas jet impinging on a liquid surface is normal. Mean drop size is increased by the introduction of bottom blowing.
\end{abstract}

KEY WORDS: drop generation; drop size distribution; combined blowing; steelmaking.

\section{Introduction}

In a recent paper, Turner and Jahanshahi' ${ }^{1)}$ reported results of mercury emulsification in a two dimensional model. The purpose of that work was to gain an insight into the drop generation phenomenon of interest to combined blowing in the BOF process. We were also motivated by a similar purpose in our work of the combined blowing process, in which water was used as the modelling liquid in both two dimensional (2-D) and three dimensional (3-D) configuration. Apart from the obvious advantage of cost and the ease of operation the use of water as the modelling liquid makes it possible to obtain the size distribution of the droplet in addition to the total quantity of the droplets ejected. The results obtained in this work, which also included high speed cinephotography, are the subject of this paper.

\section{Experimental}

The experiments were carried out in a $3-\mathrm{D}$ water model with a diameter of $200 \mathrm{~mm}$ using a set-up shown schematically in Fig. 1. The bath depth was $80 \mathrm{~mm}$. The ratio of bath height/model height was about 0.8 to allow the ejected droplets to fall outside the model. Nitrogen, metered by rotameters in the usual way, was blown through the top lance with one hole ( $2 \mathrm{~mm} \mathrm{ID)}$ or through both the top lance and bottom tuyeres with diameter of $3 \mathrm{~mm}$, at various controlled gas-flow rates. As a large amount of water is ejected out of the bath during blowing, the water level was maintained at a constant height by connecting the model with a flexible tube to a large water reservoir placed on an electronic balance, which was set to zero before each run started. In these experiments, only the total amount of splash ejected to the outside of the model was considered. After blowing, water was poured into the reservoir until the reading of the balance returned to zero. The weight of the water poured into the reservoir was recorded as the total amount of droplets ejected. The amount of the splash was divided by blowing time to obtain the rate of drop generation. In these experiments, each run was repeated until relative error of results was less than $5 \%$. Average value of the results was plotted in figures. In the experiments of droplet size distribution, a liquid nitrogen bath was placed beside the model to collect the droplets blown out of the model. The frozen droplets were analysed by using a set of sieves.

In order to explain the mechanism of the drop generation and the effect of bottom blowing on the droplet generation, high speed film was taken (5000 frames/s) using a 2-D water model $(300 \times 200 \times 20$ $\mathrm{mm}$ ). A lance with a rectangular hole of $11.5 \times 0.16$ $\mathrm{mm}$ was used in these experiments.

In the present work the slag phase was not used. The reasons for this, apart from experimental simplicity and convenience, are as follows. From observation of high speed film taken in two-phase (water/ paraffin oil) model, it was found that when a jet

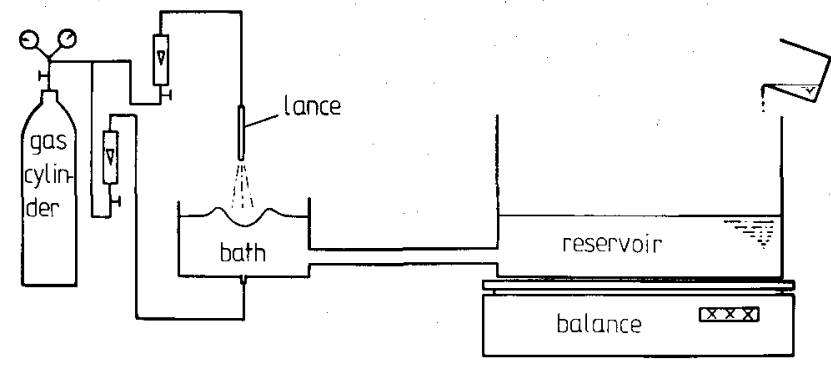

Fig. 1. Line diagram of experimental apparatus. 
impinges on the slag (oil) surface the jet pushes the slag outwards and interacts with the bath directly at the impingement zone where the droplets are generated. The bath is exposed to the jet all the time during blowing if the jet momentum is high enough. Based on this observation, the same mechanism of metal drop generation due to an impinging jet would be expected in single and two phase cases. Additionally, preliminary experiments with a two-phase (mercury/glycerin) system in which $\mathrm{Hg}$ content of the mercury/glycerin emulsion was explored, showed the same pattern in the results as in the single-phase experiments.

\section{Results and Discussion}

As with the movement of any substance, the phenomenon of droplets ejected by a gas jet impinging on a liquid surface must obey the law of mechanics. A theoretical explanation of the droplet generation, based on a force balance, was proposed by Kleppe and Oeters. ${ }^{2}$ This model considers a depression forming in the liquid surface due to the momentum of the gas jet. The deflected gas flowing along the surface of the depression exerts a shear force on the surface and drives the surface liquid flow. Because of the action of centripetal forcc caused by gravity and surface forces, the surface liquid flowing at a certain velocity tends to curvilinear movement. Droplet ejection from the bath depends on the balance of forces exerted on it. It is known that there are no droplets produced at the edge of the crater when jet momentum is very low. Because the dense phase has a tendency of self-adjustment by changing the shape of the depression to keep the force balance on the droplet, that is, the centripetal force required for the surface liquid to tend to curvilinear motion is always equal to that exerted on the surface liquid by changing the radius of curvature of the depression surface in this region. Once the required centripetal force becomes larger, due to further increase in the gas flow rate, than the exerting centripetal force, droplets will be generated at the edge of the depression. According to above analysis, it can be concluded that, for a certain system, any factor which is able to increase the shear force being exerted on the crater surface and/or the velocity of surface liquid can increase the rate of droplet generation.

\subsection{Droplet Generation}

\subsubsection{Top Blowing}

Fig. 2 shows the variation of the rate of droplet generation with top gas flow rate. It is evident that the drop generation rate greatly increases with increasing the top flow rate. Two regions of the top gas flow rate, according to the droplet generation, have been found from the results. The rate of drop generation increases with the gas flow rate slower at low flow rate $(\mathrm{AB})$, than at high flow rate (BD).

To gain an insight into the mechanisms behind this phenomenon, high speed films were taken under varying top blowing conditions. Two mechanisms of the droplet generation, corresponding to the two regions in Fig. 2, were identified from the observation of the high speed films. When the top gas flow rate is low a shallow depression with small ripples in the surface forms. In this case, a single droplet gradually forms at the surface of the crater and finally departs at the edge of the crater (see Fig. 3(a)). The generation of individual droplets is a characteristic of this range of top flow rate, which corresponds to the line $A B$ in Fig. 2, called "dropping" region in this paper.

Further increase of the top flow rate leads to the change of the mechanism of drop generation. In this region, not only single droplets but also large tears of liquid are produced at the edge of the crater during blowing. Increase of the top flow rate results in the growth of the ripples in the crater surface. Each of the ripples might cause the ejection of liquid tears at the edge of the crater. From observation of the high speed films, it has been found that formation of a tear of liquid starts with a ripple which becomes bigger and bigger as it moves up along the crater surface. Finally, a necking-off forms due to the ripple, and the liquid tear is cut off at the edge of the crater (see

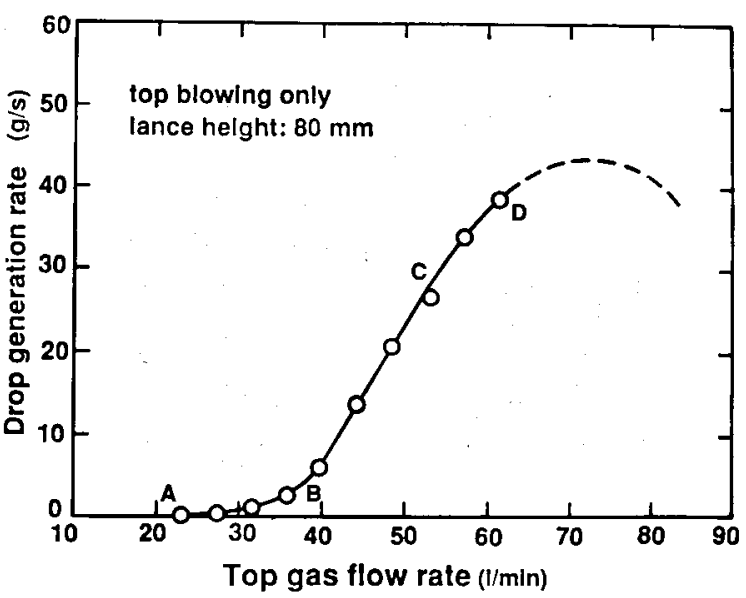

Fig. 2. Effect of gas jet momentum on drop generation rate (3-D).
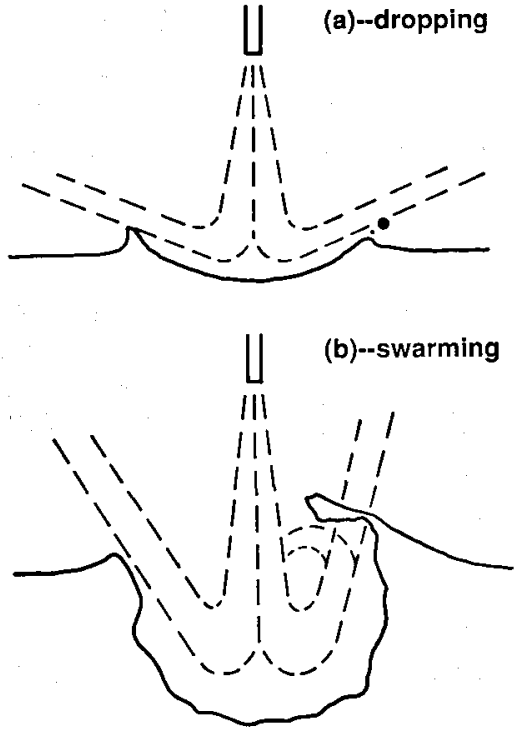

Fig. 3. The two regions of drop generation (2-D). 
Fig. 3(b)). The liquid tear is impacted by the deflected gas flow to become several small drops. Meanwhile, a number of individual drops are directly generated from the crater. The generation of liquid tear is a characteristic of this range of top flow rate, which corresponds to the line BD in Fig. 2, called "swarming " region in this paper. The reason why there is different increasing rate of drop generation in "dropping " region and in the "swarming " region is that different mechanisms of drop generation exist in different regions.

It should be pointed out that the two modes, viz., splashing and penetrating, defined in Ref. 3) and based on the flow pattern of a gas jet in the impingement zone, are different from the two regions defined in this paper on the basis of the mechanisms of the drop generation.

From Fig. 2 it can be seen that the increase in the rate of the droplet generation with increasing top flow rate tends to be slower when gas flow rate is over a certain value (point $C$ in Fig. 2). This phenomenon may be explained as follows: a proportion of the splash ejected from the bath is entrained by the gas jet and then returned to the bath, when top flow rate is over a certain value, as has been found in the present investigation, and also referred to by Molloy. ${ }^{3}$ ) The data which were used in the present experiments to obtain the droplet generation rate were the mass of the dense phase which was ejected and fell outside of the model vessel. The drop generation rate shown in Fig. 2 is lower than the actual rate. As the proportion of dense phase entrained increases with increasing top flow rate, the slope of the tangent of curve CD decreases as the top flow rate increases.

Due to the safety limit of the equipment used with respect to high pressure, variation of the drop generation rate with further increase in top gas flow rate (over $66.25 \mathrm{l} / \mathrm{min}$ ) was not investigated in the present work. However, based on the results in Fig. 4 which show the variation of the drop generation rate with lance height and that maximum generation rate is obtained at a certain lance height, it is reasonable to expect that the drop generation rate would vary along

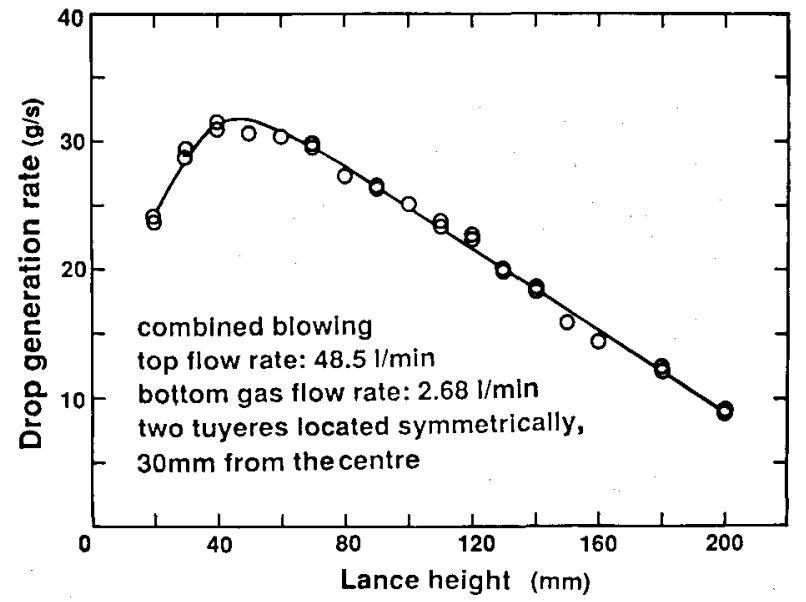

Fig. 4. Variation of drop generation rate with lance height (3-D). the dotted line in Fig. 2 with further increase of the gas flow rate. This is because the increase in gas flow rate is, to a great extent, equivalent to the decrease in the lance height.

\subsubsection{Combined Blowing}

Fig. 5 shows the effect of bottom blowing on the drop generation in the combined blowing. The rate of droplet generation is greatly increased by introduction of bottom blowing. Initially, droplet generation is proportional to bottom gas flowrate. A plateau is reached after the bottom flowrate is increased to the value of about $6 \mathrm{l} / \mathrm{min}$. No increase in the rate of drop generation was obtained with further increasing the bottom flowrate. This result is therefore the same as that reported by Turner and Jahanshahi ${ }^{11}$ for a 2dimensional mercury-glycerine model. In the present investigations, it has been found that the significant increase in the droplet generation rate is caused by interaction between bottom blowing and top blowing in the impingement zone, but not by bottom blowing itself.

The bottom blowing must influence the droplet generation in two ways, viz., by a direct effect and by an indirect effect. The direct effect means the direct interaction between the bottom gas bubbles and the impingement zone when the bubbles rise up and pass the impingement zone. On the other hand, during ascent the bubbles apply the energy due to buoyancy into the bath and accelerate it. The change of flow pattern due to the bottom blowing must influence the fluid flow at the surface of the crater, which affects the droplet generation as mentioned above. This is the indirect effect referred to in this paper.

A mechanism for the effect of bottom blowing on the drop generation is shown in Fig. 6 (single bottom tuyere located centrally). From Fig. 6, it is clearly seen that a bubble floats up and turns near the bottom of the crater and then travels up along the crater surface. The bubble then bursts near the free surface of the bath, which causes a large ripple in the surface. The extra ripples, relative to the intrinsic ones caused by top blowing only, result in increased splash from the bath. From Fig. 6, it is also clearly seen that the ripple due to the bursting of the bubble, becomes a necking-off that causes a liquid tear and a few drops

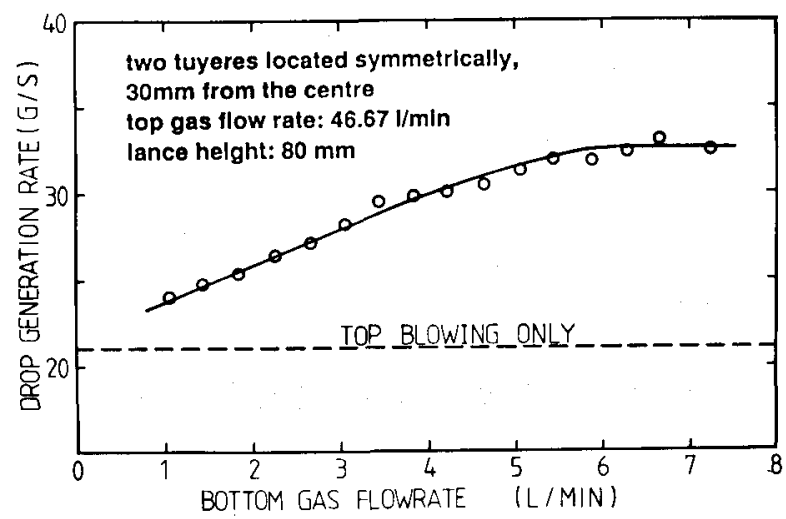

Fig. 5. Variation of drop generation rate with bottom gas flow rate (3-D). 

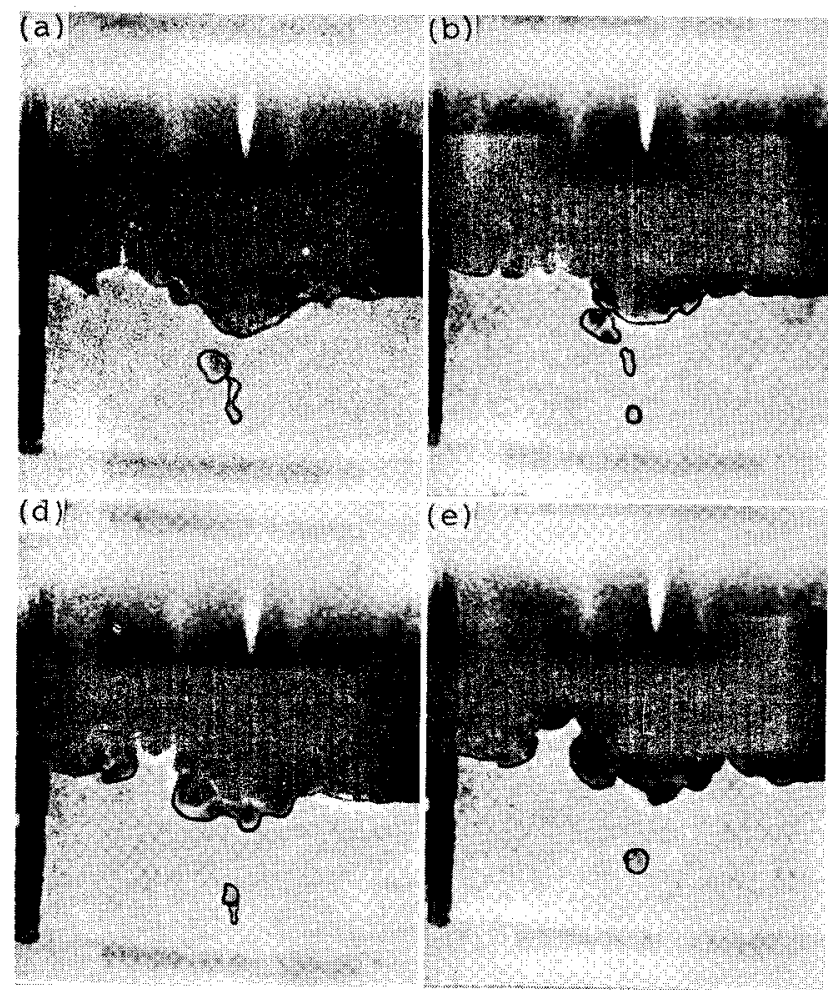

(e)

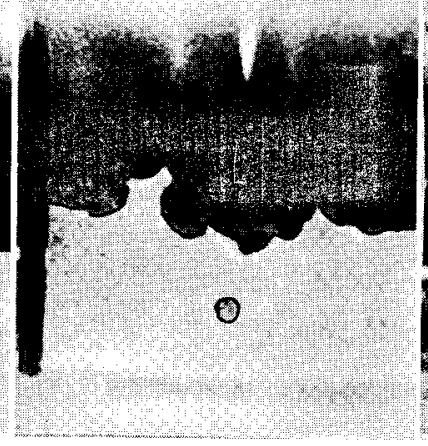

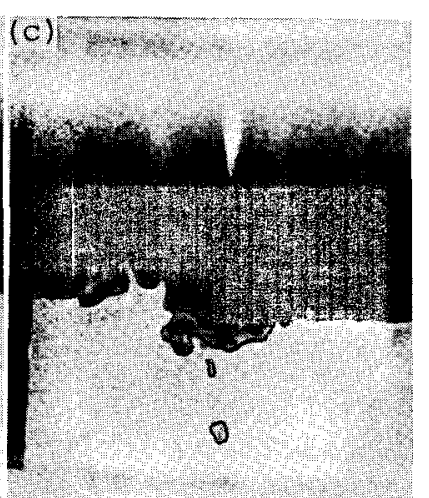

$(\mathrm{C})$

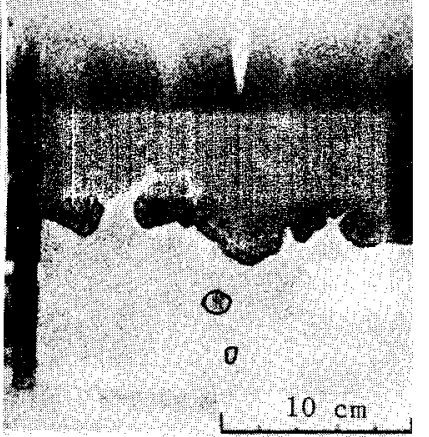

(a) $t=0.0 \mathrm{~s}$

(c) $t=0.055 \mathrm{~s}$

(b) $t=0.04 \mathrm{~s}$

(e) $t=0.085 \mathrm{~s}$

(d) $t=0.07 \mathrm{~s}$

Gas flow rates: $26.0 \mathrm{l} / \mathrm{min}$ (top) and $1.62 \mathrm{l} / \mathrm{min}$ (bottom)

Lance height: $80 \mathrm{~mm}$ bottom tuyere located at centre

Fig. 6.

Interaction between top and bottom blowing (2-D). to be produced. This phenomenon can not occur in the case of bottom blowing only, i.e., it is the direct effect of bottom blowing.

Additionally to the above, there are also some open bubbles at the bottom of the crater when the introduction of bottom blowing is central. When the bubble is opened up, it becomes a part of the crater surface, which increases the surface area of the crater, but the bubble does not cause a large ripple. In this case, the influence of the bottom blowing on the droplet generation is by the indirect effect, the efficiency of which is much smaller than that of the direct effect. Therefore, the effect of the bottom blowing on the drop gneration depends, to a great extent, on the proportion of the bubbles which float up along the side of the crater to the total number of the bubbles which are blown into the bath through the bottom tuyeres. The higher this proportion is, the greater is the effect of the bottom blowing on the drop generation. From the observation of high speed films, for low top gas flowrate, most of the bubbles turn near the bottom of the crater, and then rise up along the side. For high top flowrate, most of the bubbles are opened up at the bottom of the crater because of the impact of a high velocity jet. These phenomena indicate that the effect of the bottom blowing on the droplet generation, relative to top blowing only, is greater for lower top gas flowrate than for higher top flowrate.

In the case when bottom tuyeres are symmetrically placed underneath the impingement zone, all of the bubbles will rise up along the side of the crater because they do not contact the bottom of the crater. Consequently, more droplets should be produced in this case, which has been confirmed in the present investigation (see Fig. 7).

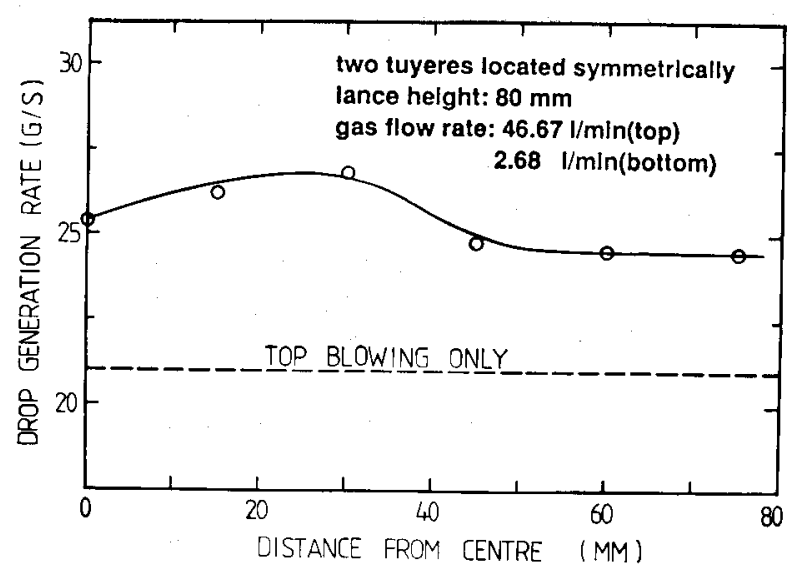

Fig. 7. Variation of drop generation rate with the position of tuyeres (3-D).

The variation of the droplet generation rate with the position of bottom tuyeres is shown in Fig. 7. In this case two tuyeres were symmetrically located at a distance away from the centre and, by inspection of Fig. 7, maximum generation rate was obtained at the distance of about $25 \sim 30 \mathrm{~mm}$. Similar results have also been reported in the 2-D mercury system referred to earlier. ${ }^{1)}$ Further increase of the distance beyond the maximum point lowers the droplet generation because of the smaller influence of the bottom blowing on the impingement zone. When the tuyeres are positioned outside the impingement zone the bottom blowing only influences the droplet generation by the indirect effect, in which case the rate of droplet generation is lower than in all cases when tuyeres are located underneath the impingement zone (see Fig. 7).

A significant conclusion that water can be used to model molten steel with respect to droplet generation 
by a jet impinging on a liquid surface may be drawn by comparing the results of the water model with those of the mercury-glycerine model. As discussed above, despite large differences of properties between water and mercury $\left(\rho_{\mathrm{II}_{2} \mathrm{O}}=1000 \mathrm{~kg} / \mathrm{m}^{3}, \rho_{\mathrm{Hg}}=13600\right.$ $\mathrm{kg} / \mathrm{m}^{3}, \sigma_{\mathrm{H}_{2} \mathrm{O}}=0.0728 \mathrm{~N} / \mathrm{m}, \sigma_{\mathrm{Hg}}=0.47 \mathrm{~N} / \mathrm{m}$ ) and the use of different dimensional models, very similar results on the droplet generation have been obtained in both models. This indicates that the results from the present investigation can be applied to explain the phenomena occurring in the basic oxygen steelmaking process.

\subsection{Drop Size Distribution}

According to the emulsion mechanism, high refining rate of $\mathrm{BOF}$ process is due to extremely large reaction interfacial area between metal droplets and slag. The interfacial area depends on two factors, viz. the number of metal droplets and drop size distribution. So one is more interested in knowing both factors than only one of them. The former was studied in this work and in Ref. 1) by considering the amount of splash from the bath. A few papers on the drop size distribution in top blowing process have been published.4,5) However, the effect of bottom blowing on the drop size distribution has not been investigated. In this work, droplet size distribution in both top blowing and combined blowing were studied.

The results of drop size distribution from the present work are shown in Figs. 8 to 12, in which cumulative weight percent remaining on the sieve against the lower limit of class diameter is plotted on a normal probability scale. Results in Figs. 8 to 10 were obtained from the 2-D model and results in Figs. 11 and 12 were obtained from the $3-D$ model. From these figures it is evident that a strong linear relationship exists, which means that the drop size distribu-

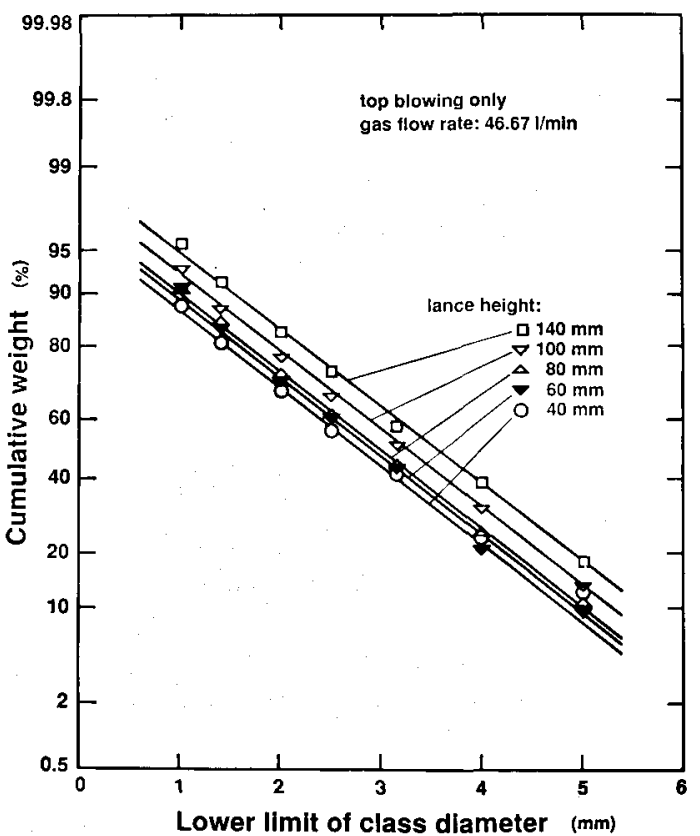

Fig. 8. Drop size distribution at different lance heights (2-D). tions are normal. The present results are identical to those of an earlier work ${ }^{4}$ where drops were generated by the breakup of a vertically falling single drop of $\mathrm{Fe}-5 \% \mathrm{C}$ due to the action of a horizontal gas jet of high velocity. But another study ${ }^{5)}$ of the drop size distribution, resulting from drops ejected from a molten bath and falling onto a platform outside the crucible, concluded that the Rosin-RammlerSperling distribution is obeyed. It should be pointed out that in the latter work, ${ }^{5}$ metal pieces which were directly ejected from the bath as well as agglomerates of drops or small metal pieces formed on the platform were involved. The size of the metal pieces involved was characterised by the equivalent spherical diame-

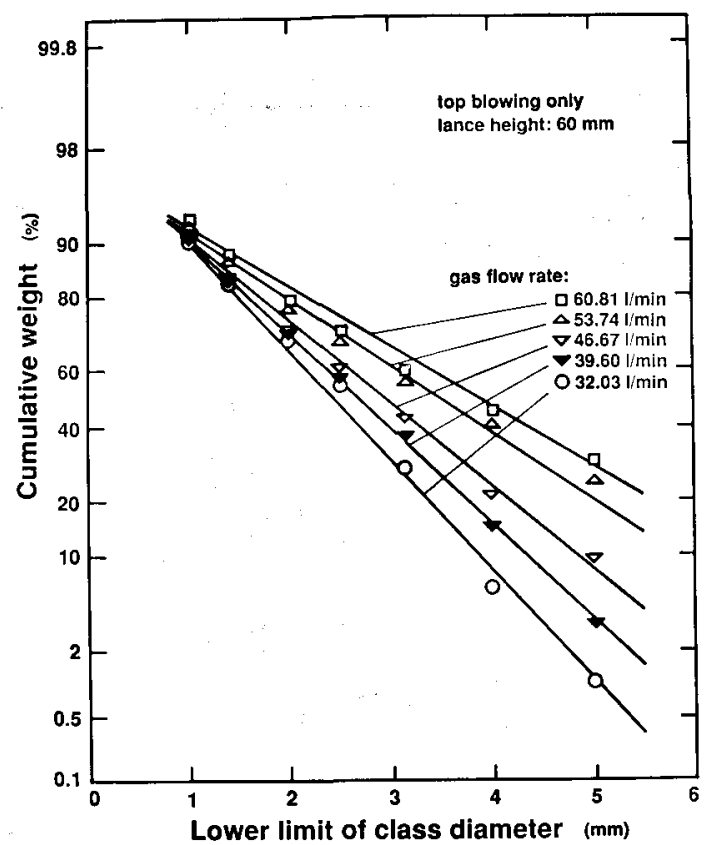

Fig. 9. Variation of drop size generation with top gas flow rate (2-D).

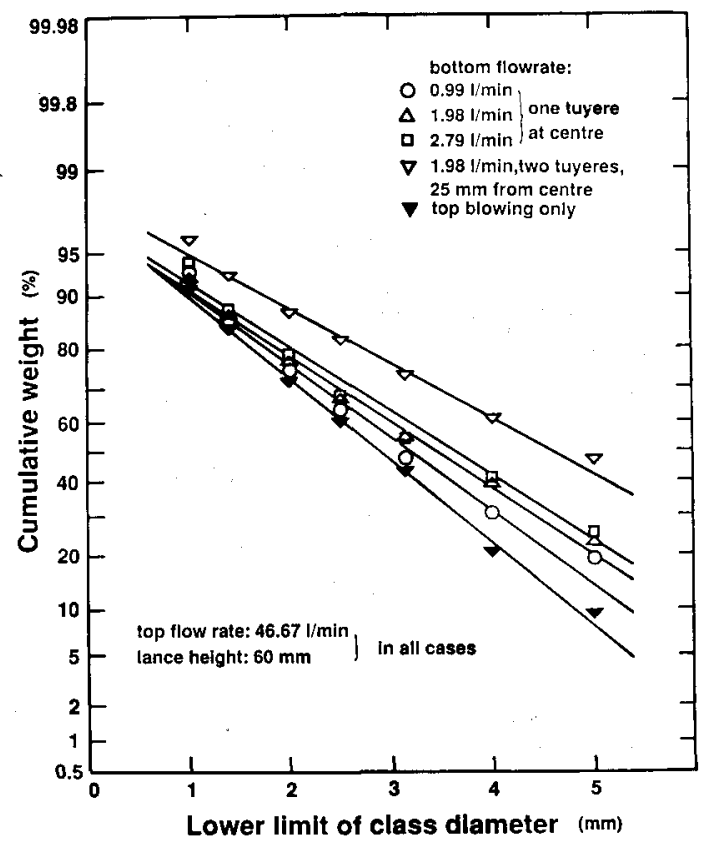

Fig. 10. Effect of bottom blowing on drop size distribution (2-D). 


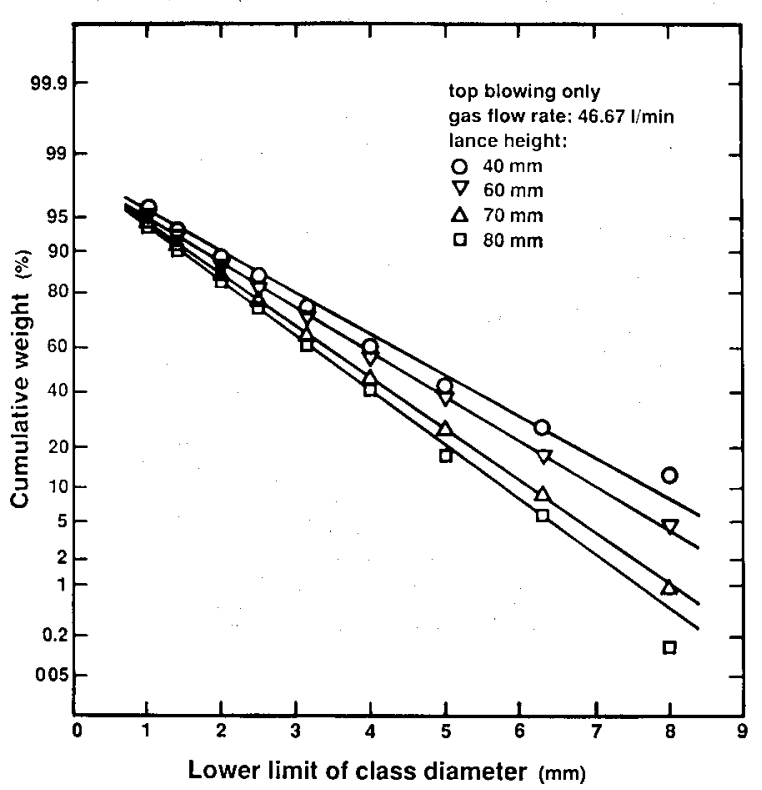

Fig. 11. Drop size distributions at different lance heights (3-D).

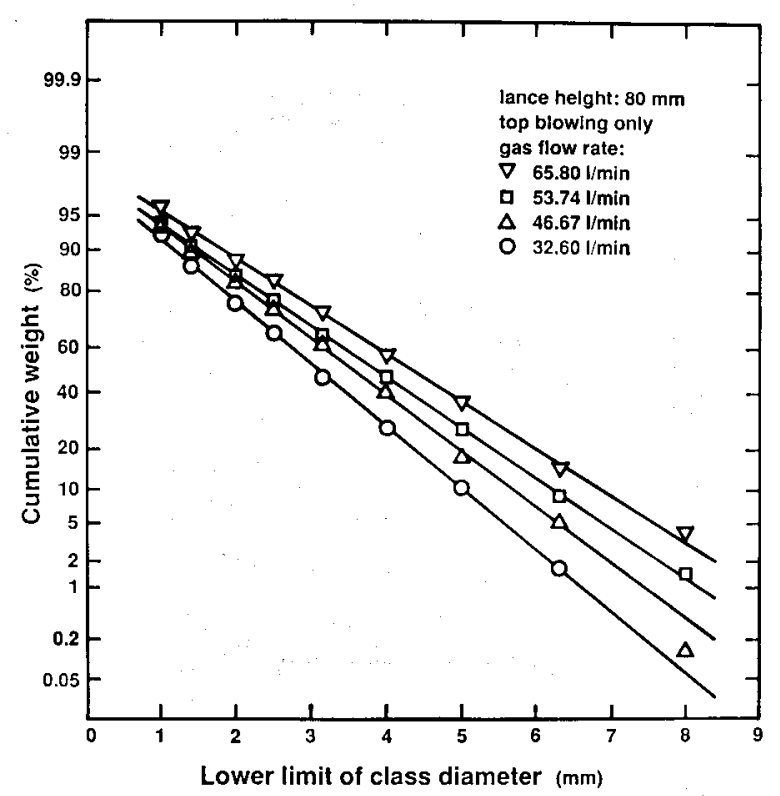

Fig. 12. Drop size distributions at different top gas flow rates (3-D).

ter. Additionally, as a very small crucible was used in that work, the flow pattern in the bath would be quite different and consequently affect the drop generation. These combined factors would almost certainly be expected to result in a different drop size distribution.

Figs. 8 and 11 show the effect of lance height on the drop size distribution, and Figs. 9 and 12 give the results of the drop size distributions at different top gas flow rates. With the exception of the results in Fig. 8, it can be seen that the proportion of large droplets increases with increasing top gas flow rate and with decreasing lance height, in other words with increasing jet momentum at the bath surface. The exception in Fig. 8 can be explained if the data are replotted in terms of the drop generation rate as a

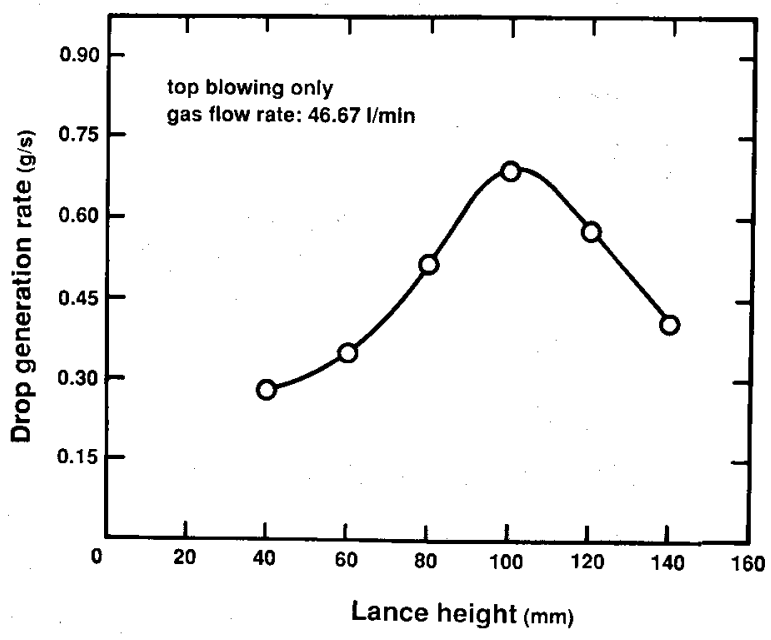

Fig. 13. Variation of drop generation with lance heights (2-D).

function of lance height as shown in Fig. 13. From Fig. 13 it can be seen that the drop generation rate increases with decreasing lance height when the lance height is large. The maximum rate is reached at the lance height of about $100 \mathrm{~mm}$ and then the drop generation decreases with further decrease of the lance height. Visual observations of the bath surface showed that when lance height is reduced down to near $100 \mathrm{~mm}$, very deep penetration forms in the bath surface and a large proportion of large size droplets is entrained by the jet and returned to the bath, and the more so the lower the lance height. This phenomenon results in the decrease in the amount of the splash and the proportion of large droplets. That is why the mean drop size decreases with decreasing lance height in Fig. 8. It should also be pointed out that the absolute values of the data in Figs. 4 and 13 are not comparable as they were obtained in 3-D and 2-D models, respectively.

From Fig. 10, it can be seen that the introduction of bottom blowing leds to the increase in the mean size of the droplets. This result may be explained by the "ripple theory" discussed earlier. The ripples on the surface of the crater caused by bottom blowing might result in liquid tears at the edge of the crater and therefore also large droplets. The increased proportion of large droplets due to the bottom blowing would decrease the effect of the bottom blowing on steelmaking refining rate than may be expected on the basis of the droplet generation rate alone as discussed above.

\section{Conclusions}

The investigation into the drop generation by a gas jet impinging on a liquid surface has indicated that there are different mechanisms of drop generation in "dropping" and "swarming" regions, which result in different increase of drop generation rate with top gas flow rate in the two regions.

The results have shown that bottom blowing significantly increases the rate of droplet generation, and that this increase is caused by the interaction of the 
bottom blowing and top blowing in the impingement zone, and not by the bottom blowing as such.

Drop size distribution generated by a gas jet impinging on a liquid surface can be satisfactorily represented by a normal distribution. Mean drop size is increased by introduction of bottom blowing.

\section{Acknowledgements}

We wish to thank Dr. G. R. Belton and Dr. C. Dobson in Central Research Laboratories of BHP Steel International Group for helpful discussion, Mr. C. L. Carey and his colleagues in the Steelmaking Technical Group of the Slab and Plate Products Division for their assistance, and Mr. K. Preuhs of Central
Laboratories for carrying out high speed photography. Financial support by BHP Steel International is also gratefully acknowledged.

\section{REFERENCES}

1) G. Turner and S. Jahanshahi: Trans. Iron Steel Inst. Jpn., 27 (1987), 734.

2) W. Kleppe and F. Oeters: Arch. Eisenhüttenwes., 48 (1977), 139.

3) N. A. Molloy: J. Iron Steel Inst., 206 (1970), 943.

4) S. C. Korja and K. W. Lange: Ironmaking Sleelmaking, 10 (1983), 160.

5) S. C. Korja and K. W. Lange: Metall. Trans. B, 15B (1984), 109. 